Article

\title{
Effects of Post-Thinning Precipitation on Soil Acid Phosphomonoesterase Activity in Larix principis-rupprechtii Mayr. Plantations
}

\author{
Huixia Tian $\mathbb{D}^{\mathrm{D}}$, Xiaoqin Cheng and Hairong Han * $\mathbb{D}$ \\ Beijing key laboratory of forest resources and ecosystem processes, Beijing Forestry University, \\ Beijing 100083, China \\ * Correspondence: hanhr6015@bjfu.edu.cn; Tel.: +86-10-6233-6015
}

Received: 26 July 2019; Accepted: 23 August 2019; Published: 26 August 2019

check for updates

\begin{abstract}
Soil phosphorus (P) is one of the essential macronutrients for plant growth. Phosphatase-mediated $\mathrm{P}$ mineralization in particular is critical for the biogeochemical cycling of $\mathrm{P}$, and its activity reflects the organic $\mathrm{P}\left(\mathrm{P}_{\mathrm{o}}\right)$ mineralization potential in soils. In recent years, global climate change has led to changes in precipitation, which inevitably has affected the P cycle as well. To study these effects of precipitation on soil acid phosphomonoesterase (AcPME) activity, the following combined thinning and precipitation treatments were conducted across Larix principis-rupprechtii Mayr. plantations in China: control (CK), light (LT), moderate (MT), and high thinning (HT). The precipitation treatments included natural precipitation (NP), 30\% reduced precipitation (RP30), and $60 \%$ reduced precipitation (RP60). Soil moisture, microbial biomass carbon (MBC), and soil P fractions were also determined to link their effects on soil AcPME. The results show that soil AcPME activity was significantly higher in the rainy season, which is associated with higher microbial activity and increased P demand, than in the dry season. Generally, soil AcPME activity was found to increase with thinning intensity. In the dry season, the NP treatment was more conducive to improving soil AcPME activity. In the rainy season, the RP60 treatment inhibited soil AcPME activity under all thinning treatments. The RP30 treatment was only found to offer a significant boost for MT. These results indicate that the potential transformation rate of $P_{0}$ may be more dependent on water in the dry season than in the rainy season. If drought occurs, the $P_{0}$ mineralization rate would decrease for all L. principis-rupprechtii plantations, but excessive rainfall in the rainy season would also impact the turnover of $\mathrm{P}_{\mathrm{o}}$ into MT adversely.
\end{abstract}

Keywords: precipitation; thinning; acid phosphomonoesterase; Larix principis-rupprechtii Mayr

\section{Introduction}

Soil phosphorus $(\mathrm{P})$ occurs in both inorganic $\left(\mathrm{P}_{\mathrm{i}}\right)$ and organic $\left(\mathrm{P}_{\mathrm{o}}\right)$ forms, and consists of compounds with varying degrees of solubility and bioavailability [1]. Most soil $\mathrm{P}$ is bound in detritus as $\mathrm{P}_{\mathrm{O}}$ and remains inaccessible to plants. Apart from the weathering of parent material in soils [2,3], P input depends on the mineralization of soil organic matter [4,5], soil-nesting insect pollinators [6], and soil-dwelling arthropods [7], who alter soil microbial communities and their function associated with P mineralization by altering frass and waste inputs, and by selectively consuming plant materials. In addition, $P$ inputs depends on the plant chemistry and their chemical composition [8]. Phosphatases, which originate from fungi, bacteria, and root exudates, catalyze the hydrolysis of ester bonds between phosphate and carbon compounds in organic substrates to enhance P availability to ecosystems $[9,10]$. The production of phosphatase increases when the demand for P increases in forest ecosystems [11]. These enzymes therefore play an important role in maintaining and controlling the rate of $\mathrm{P}$ cycling in 
forest ecosystems [12]. Of the phosphatases, phosphomonoesterases are the most widely studied [13]. Phosphomonoesterase activity provides useful information on the $\mathrm{P}_{\mathrm{o}}$ mineralization potential and biological activity in soils [14]. Usually, acid phosphomonoesterase (AcPME) prevails in acidic soils whereas alkaline phosphomonoesterase prevails in alkaline soil [15].

Global climate change models have predicted that precipitation patterns will undergo major changes in certain geographic regions in the second half of the 21st Century. In particular, precipitation is expected to decrease in northern China [16]. These predicted changes will greatly alter the soil moisture dynamics [17], which may in turn affect the dynamics of the soil $\mathrm{P}$ cycle [18,19]. Soil moisture is a key factor controlling soil $\mathrm{P}$ availability via several processes including $\mathrm{P}_{\mathrm{o}}$ mineralization [20], the demand of $\mathrm{P}$ for plant growth, and microbial activity [12,21]. For example, in the Mediterranean region, soil phosphatase activity was reduced during an increasingly strong drought and the $\mathrm{P}$ available for uptake by plants also decreased, which resulted in a significant limitation for plant growth [22,23]. Sardans and Peñuelas [24] found that a $21 \%$ reduction of soil moisture resulting from runoff and rainfall exclusion lowered AcPME activity by $31 \%-40 \%$. Similarly, a six-year drought manipulation experiment where soil moisture was lowered by $13 \%$ to $29 \%$ showed that water stress results in reduced microbial activity; soil AcPME activity was lowered by $22 \%$ to $27 \%$. At the same time, drought augmented the availability of organic extractable $P$ [25]. Therefore, changes in precipitation are likely to affect the rate of $\mathrm{P}$ turnover and soil $\mathrm{P}$ availability, but droughts may offer benefits too. Because soil AcPME is associated with $\mathrm{P}$ cycling in forest ecosystems, it is a good measure of how changes in precipitation affect the P supply overall [26]. However, to our best knowledge, the effects of precipitation on soil AcPME activity have been unstudied in warm temperate forests. Understanding how soil phosphatase activity responds to drought events will help inform understanding of future soil dynamics and aid in developing proper silvicultural guidelines in this new global change context [27].

In the warm temperate zone, Larix principis-rupprechtii Mayr. is one of the typical afforestation species [28]. The high initial planting density and the management lag have resulted in incredibly dense canopies in existing stands, which reduces soil fertility and forest quality with low yield and efficiency [29]. Reasonable thinning can promote improve the light, water, temperature and soil nutrient availability conditions in forests, thereby improving forest productivity [30]. These L. principis-rupprechtii plantations provide an excellent opportunity to study soil P cycling at different thinning intensities. Previous studies have shown that soil $\mathrm{P}$ availability varies with the degree of thinning, with maximum P availability under moderately thinned (MT) plantantions [31]. In this study, we aim to study how soil AcPME activity responds to precipitation in plantations having different $P$ availabilities.

We conducted a field experiment to apply various precipitation treatments (natural precipitation (NP), 30\% reduced precipitation (RP30) and 60\% reduced precipitation (RP60) )across L. principisrupprechtii plantations following different thinning regiments to test the hypotheses: (1) Regarding the influence of seasonal precipitation, soil AcPME activity will be greater during the rainy season than the dry season; (2) Plantations having different thinning regiments will differ in their response to the precipitation treatments, but soil AcPME activity will be greatest in MT plantations; (3) Precipitation treatments will have different effects on soil AcPME activity during the dry and rainy seasons.

\section{Materials and Methods}

\subsection{Site Description and Experimental Design}

The study was conducted at Mt. Taiyue in Shanxi, North China $\left(36^{\circ} 31^{\prime}-36^{\circ} 43^{\prime} \mathrm{N}, 112^{\circ} 01^{\prime}-112^{\circ} 15^{\prime}\right.$ E; elevation 2273-2359 m.a.s.l.). The area has a semi-humid temperate monsoon climate, consisting of cold, dry winters and hot, rainy summers. The mean annual temperature is about $8.7^{\circ} \mathrm{C}$, with a minimum monthly average temperature of $-10.4{ }^{\circ} \mathrm{C}$ in January and a maximum monthly average temperature of $17.4^{\circ} \mathrm{C}$ in July. The average frost-free period lasts 125 days, with an early frost in October and a late frost in April. The mean annual precipitation ranges from $600 \mathrm{~mm}$ to $650 \mathrm{~mm}$, of which 
more than $60 \%$ falls from May to October (rainy season) and less than 40\% falls between November and April (dry season). The soil type is Alfisol, according to the U.S. soil classification system [32]. The zonal vegetation is a temperate deciduous broad-leaved forest, and the dominating tree species are Betula platyphylla Suk., Quercus wutaishanica Mayr., Pinus tabuliformis Carr. and L. principis-rupprechtii.

In spring 1982, several three-year-old L. principis-rupprechtii seedlings were planted at a density of 3000 trees $\mathrm{ha}^{-1}$. The seedlings were planted along contour lines in the mountainous areas of the bush vegetation, following forestry guidelines used to meet timber demands. In April 2010, thinning management treatments were carried out for entire stands on L. principis-rupprechtii plantations to lower the density to 2160 trees ha ${ }^{-1}$. After thinning, twelve $25 \mathrm{~m} \times 25 \mathrm{~m}$ plots were set up at a distance of at least $10 \mathrm{~m}$ apart in order to avoid edge effects. In March 2012, the twelve plots were randomly assigned to four distinct treatments of secondary thinning: light thinning (LT, thinned to 1835 trees ha ${ }^{-1}$ ), moderate thinning (MT, thinned to 1413 trees ha ${ }^{-1}$ ), high thinning (HT, thinned to 1086 trees ha ${ }^{-1}$ ), and the control (CK, unthinned). Thus, this study was conducted in a completely randomized design with three replications per treatment. Detailed information on these four thinning treatments can be found in Table S1 [31].

In April 2016, within each of the 12 plots, we randomly established three precipitation treatments, including natural precipitation (NP), 30\% reduced precipitation (RP30), and $60 \%$ reduced precipitation (RP60) with three replicate subplots $(3 \mathrm{~m} \times 3 \mathrm{~m}$ ) for each treatment. In the reduced precipitation (RP) subplots, a $3.5 \mathrm{~m} \times 9 \mathrm{~cm}$ concave transparent polyvinyl chloride (PVC) plate was evenly installed over which a rain reduction frame was made in series with iron wire to intercept the precipitation. The gap between the transparent plates of the RP30 subplot was $21 \mathrm{~cm}$, while the gap for the RP60 subplot was $6 \mathrm{~cm}$. The rain reduction frame was placed $\sim 80-100 \mathrm{~cm}$ above the ground to prevent rainwater from entering the subplots, and the corresponding area of the subplot was uniformly covered to form a rainproof surface. To prevent surface runoff and lateral movement of moisture in the surrounding soil, thick PVC plates were inserted into the ground to a depth of $15 \mathrm{~cm}$ around each RP subplot. The control subplots, receiving natural precipitation, were built beside these RP subplots. The litter that fell onto the rain reduction frames of RP subplots was removed manually and periodically sprinkled onto the soil below the plates.

\subsection{Soil Sampling}

We conducted soil sampling in April (the dry season) and August (the rainy season) of 2018. Nine soil cores (three random points per subplot from each three replicate subplots) at $0-10 \mathrm{~cm}(2.5 \mathrm{~cm}$ diameter) were collected for each precipitation treatment in each plot and mixed to form a composite soil sample. Soil samples transferred into sealed plastic bags and immediately taken to the laboratory. After removing roots and plant residues, the composite sample was sieved through a 2-mm mesh sieve and divided into two parts. One was immediately stored at $4{ }^{\circ} \mathrm{C}$ for measuring soil AcPME activity and microbial biomass carbon (MBC). The other was air-dried and further sieved to $0.25 \mathrm{~mm}$ for chemical property analysis of soil total carbon (TC), total nitrogen (TN) and P fractionation, and through a 2-mm sieve for soil $\mathrm{pH}$ analysis.

\subsection{Soil Analysis}

Soil moisture of the top $10 \mathrm{~cm}$ was measured twice a month within a subplot using a Theta Probe ML2X with an HH2 moisture meter (Delta-T Devices, Cambridge, England). Soil pH value was measured in an aqueous extract (1:2.5 soil:water) with a pH-Meter (pH-10, Sartorius, Göttingen, Germany). Soil TC and TN were determined by dry combustion with an elemental analyzer (FLASH2000 CHNS/O, Thermo, Third Avenue, Waltham, MA, USA). Soil MBC was determined using the chloroform fumigation extraction technique [33]: a 5-g quantity (dry weight equivalent) of soil was fumigated for $24 \mathrm{~h}$ at $25^{\circ} \mathrm{C}$. Organic $\mathrm{C}$ from fumigated and non-fumigated (control) soil samples extracted with $0.5 \mathrm{M} \mathrm{K}_{2} \mathrm{SO}_{4}$ and quantified with a total organic carbon analyzer (Multi N/C 3000, Analytik Jena AG, Konrad-Zuse-Straße, Berlin, Germany). Then non-fumigated values were subtracted from fumigated 
values and MBC was calculated using a $\mathrm{k}_{\mathrm{EC}}$ factor of 0.45 [34]. Soil AcPME activity was assayed via the standard method of Tabatabai and Bremner [35]. Briefly, $1 \mathrm{~g}$ of fresh soil was well mixed with modified universal buffer (MUB, $\mathrm{pH}$ 6.5) and substrate ( $p$-nitrophenyl phosphate) solution and then incubated at a temperature of $37^{\circ} \mathrm{C}$ for $1 \mathrm{~h}$. Then the amount of $p$-nitrophenol release was measured spectrophotometrically.

The sequential extraction procedure developed by Hedley, et al. [36] and modified by Tiessen and Moir [37] was used to assess soil $\mathrm{P}$ fractions. In general, $0.5 \mathrm{~g}$ of air-dried soil was sequentially extracted with an anion exchange resin membrane (defined as $\mathrm{R}-\mathrm{P}_{\mathrm{i}}$ fraction), $0.5 \mathrm{M} \mathrm{NaHCO} 3\left(\mathrm{Bic}-\mathrm{P}_{\mathrm{i}}\right.$ and Bic- $\mathrm{P}_{\mathrm{o}}$ fractions), $0.1 \mathrm{M} \mathrm{NaOH}\left(\mathrm{OH}-\mathrm{P}_{\mathrm{i}}\right.$ and $\mathrm{OH}-\mathrm{P}_{\mathrm{o}}$ fractions), $1.0 \mathrm{M} \mathrm{HCl}$ (Dil.HCl- $\mathrm{P}_{\mathrm{i}}$ fraction)

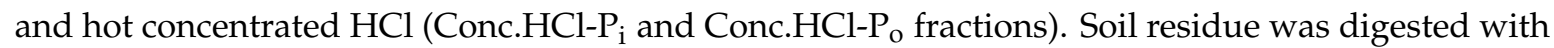
concentrated $\mathrm{H}_{2} \mathrm{SO}_{4}$ and $\mathrm{H}_{2} \mathrm{O}_{2}$ (Res- $\mathrm{P}_{\mathrm{i}}$ fraction). All extraction solutions were measured using a modified phosphomolybdate blue method [38].

\subsection{Statistical Analysis}

Both main effects and interactive effects of thinning and season on soil properties were tested using repeated-measures analysis of variance (ANOVA). Differences were examined between the dry and rainy seasons for soil moisture, soil properties and soil AcPME activity using the $t$-test for independent samples. One-way ANOVA, followed by Tukey's multiple comparisons post hoc test at $p<0.05$ level, was used to evaluate the differences in soil moisture, soil properties and soil AcPME activity among the NP treatments in types of thinned plantations; and the effects of precipitation intensity on dependent variables among the treatments separately for each season and thinning. Correlations between soil property and AcPME activity were assessed using Pearson's correlation coefficients. All data were checked for normality and homoscedasticity prior to performing the statistical analyses and were log-transformed when necessary to correct for deviations from these assumptions. P fractions were log-transferred to normalize data distribution. Analyses were performed using SPSS, version 21.0 (IBM, Chicago, IL, USA).

\section{Results}

\subsection{Soil Moisture}

In all plantations, the mean soil moisture was significantly greater in the rainy season (May to October) than in the dry season (November to April) (Figure 1). For the NP treatment, annual mean soil moisture was relatively higher in MT and HT than in LT and CK. Specifically, soil moisture increased in MT $(p=0.029)$ and HT $(p=0.048)$ in the dry season more than CK (Figure S1). The values of mean annual soil moisture across all thinning treatments were $26.1 \%$ for NP, $23.5 \%$ for RP30, and $20.5 \%$ for RP60. Compared to NP, the RP60 soil moisture decreased significantly (Figure S2). 

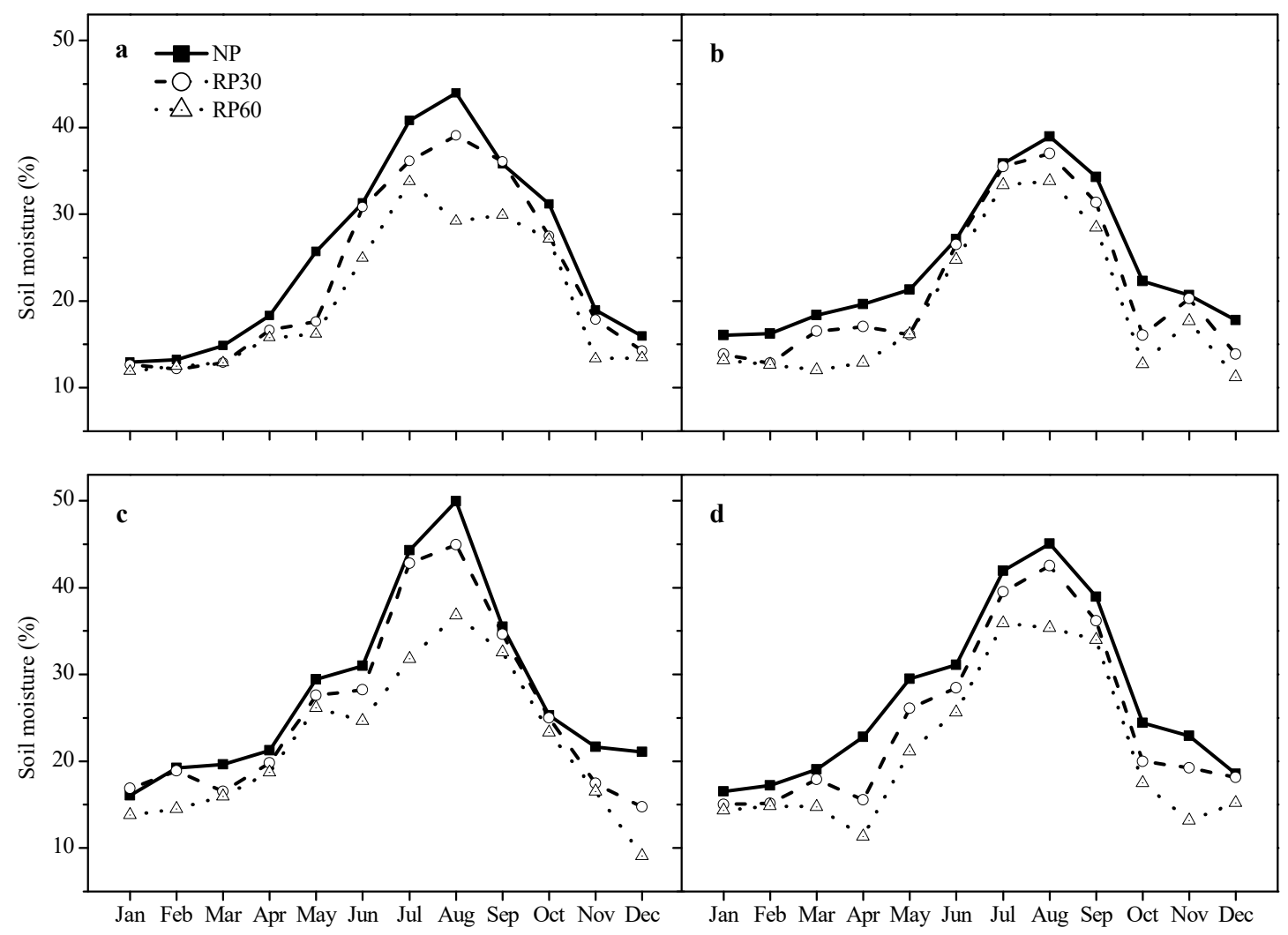

Figure 1. Seasonal dynamics of soil moisture under different precipitation treatments from January to December 2018 in the control (a), lightly thinned (b), moderately thinned (c), and highly thinned (d) L. principis-rupprechtii plantations. NP, natural precipitation; RP30, 30\% reduced precipitation; RP60, $60 \%$ reduced precipitation.

\subsection{Soil Chemical and Microbial Properties}

In the dry season, there were no significant differences in $\mathrm{pH}, \mathrm{TC}$, and $\mathrm{TN}$ among the three precipitation treatments. NP had greater TP $(p=0.021)$ and MBC $(p=0.015)$ than RP60 under CK and MT respectively (Table 1). The concentrations of Bic- $\mathrm{P}_{\mathrm{o}}$ and Conc.HCl- $\mathrm{P}_{\mathrm{o}}$ decreased as the amount of precipitation was reduced, and accordingly they were significantly higher under the NP treatment than the RP60 treatment, except for in the HT (Table 2). In the rainy season, the RP60 treatment significantly lowered soil $\mathrm{pH}$ in LT to a greater extent than the NP treatment did (Table 1). Concentrations of $\mathrm{TP}, \mathrm{Bic}-\mathrm{P}_{\mathrm{o}}, \mathrm{OH}-\mathrm{P}_{\mathrm{o}}$, and Conc.HCl- $\mathrm{P}_{\mathrm{o}}$ were almost always lower in the RP60 treatment than the NP treatment (Tables 1 and 2). The only exceptions to this trend were samples collected in MT (Bic- $\mathrm{P}_{\mathrm{o}}$ ) and HT (Conc.HCl- $\mathrm{P}_{\mathrm{o}}$ ). Most soil properties were significantly correlated with soil AcPME, except for TC $(p=0.194)$ and Bic- $P_{i}(p=0.678)$ (Table 3$)$. 
Table 1. Soil properties in the dry and rainy seasons and their responses to the various precipitation and thinning treatments in L. principis-rupprechtii plantations.

\begin{tabular}{|c|c|c|c|c|c|c|c|}
\hline Season & Thinning & Precipitation & $\mathrm{pH}$ & $\mathrm{TC}\left(\mathrm{g} \mathrm{kg}^{-1}\right)$ & $\mathrm{TN}\left(\mathrm{g} \mathrm{kg}^{-1}\right)$ & $\mathrm{TP}\left(\mathrm{g} \mathrm{kg}^{-1}\right)$ & $\mathrm{MBC}\left(\mathrm{mg} \mathrm{kg}^{-1}\right)$ \\
\hline \multirow[t]{16}{*}{ Dry } & CK & $\mathrm{NP}$ & $6.4 \pm 0.2$ & $43.7 \pm 3.7$ & $3.7 \pm 0.4$ & $0.34 \pm 0.01$ & $345.0 \pm 2.2 \mathrm{a}$ \\
\hline & & RP30 & $6.0 \pm 0.0$ & $66.1 \pm 11.7$ & $5.2 \pm 1.0$ & $0.29 \pm 0.00$ & $273.4 \pm 12.6 \mathrm{ab}$ \\
\hline & & RP60 & $6.0 \pm 0.1$ & $44.1 \pm 1.1$ & $3.4 \pm 0.1$ & $0.32 \pm 0.02$ & $231.4 \pm 31.3 b$ \\
\hline & & $p$ & 0.113 & 0.112 & 0.189 & 0.062 & 0.017 \\
\hline & LT & $\mathrm{NP}$ & $6.3 \pm 0.1$ & $38.8 \pm 2.2$ & $3.1 \pm 0.2$ & $0.41 \pm 0.03$ & $343.2 \pm 7.3$ \\
\hline & & RP30 & $5.7 \pm 0.1$ & $39.7 \pm 5.2$ & $3.7 \pm 0.1$ & $0.36 \pm 0.01$ & $349.2 \pm 108.8$ \\
\hline & & RP60 & $6.2 \pm 0.4$ & $41.0 \pm 1.5$ & $3.7 \pm 0.3$ & $0.37 \pm 0.00$ & $331.7 \pm 76.1$ \\
\hline & & $p$ & 0.290 & 0.898 & 0.196 & 0.130 & 0.987 \\
\hline & MT & $\mathrm{NP}$ & $6.1 \pm 0.2$ & $53.4 \pm 0.7$ & $4.8 \pm 0.2$ & $0.63 \pm 0.04 \mathrm{a}$ & $389.2 \pm 13.5$ \\
\hline & & RP30 & $5.9 \pm 0.1$ & $63.3 \pm 4.8$ & $5.0 \pm 0.4$ & $0.53 \pm 0.04 \mathrm{ab}$ & $355.1 \pm 23.0$ \\
\hline & & RP60 & $5.8 \pm 0.0$ & $56.8 \pm 3.3$ & $4.6 \pm 0.2$ & $0.44 \pm 0.02 \mathrm{~b}$ & $348.1 \pm 19.0$ \\
\hline & & $p$ & 0.207 & 0.188 & 0.565 & 0.025 & 0.326 \\
\hline & HT & $\mathrm{NP}$ & $6.1 \pm 0.0$ & $54.8 \pm 4.8$ & $4.9 \pm 0.5$ & $0.46 \pm 0.04$ & $383.1 \pm 14.5$ \\
\hline & & RP30 & $5.7 \pm 0.1$ & $58.0 \pm 0.8$ & $4.4 \pm 0.1$ & $0.43 \pm 0.02$ & $425.6 \pm 37.6$ \\
\hline & & RP60 & $5.8 \pm 0.1$ & $59.5 \pm 8.1$ & $4.7 \pm 0.5$ & $0.42 \pm 0.02$ & $338.5 \pm 37.2$ \\
\hline & & $p$ & 0.065 & 0.825 & 0.769 & 0.712 & 0.230 \\
\hline \multirow[t]{16}{*}{ Rainy } & CK & NP & $6.1 \pm 0.0$ & $48.2 \pm 4.0$ & $2.0 \pm 0.1$ & $0.51 \pm 0.00 \mathrm{a}$ & $649.9 \pm 12.3$ \\
\hline & & RP30 & $6.2 \pm 0.1$ & $45.1 \pm 5.0$ & $2.2 \pm 0.2$ & $0.44 \pm 0.01 b$ & $659.2 \pm 60.5$ \\
\hline & & RP60 & $6.2 \pm 0.1$ & $47.2 \pm 4.1$ & $2.0 \pm 0.2$ & $0.38 \pm 0.01 \mathrm{c}$ & $624.8 \pm 33.9$ \\
\hline & & $p$ & 0.760 & 0.878 & 0.736 & $<0.001$ & 0.831 \\
\hline & LT & $\mathrm{NP}$ & $6.1 \pm 0.1 \mathrm{a}$ & $39.4 \pm 3.1$ & $3.2 \pm 0.4$ & $0.57 \pm 0.02 \mathrm{a}$ & $537.8 \pm 5.9$ \\
\hline & & RP30 & $5.9 \pm 0.0 \mathrm{ab}$ & $45.2 \pm 1.3$ & $3.6 \pm 0.1$ & $0.47 \pm 0.02 b$ & $561.7 \pm 86$ \\
\hline & & RP60 & $5.8 \pm 0.0 \mathrm{~b}$ & $43.2 \pm 0.4$ & $3.4 \pm 0.1$ & $0.46 \pm 0.02 b$ & $650.7 \pm 41.7$ \\
\hline & & $p$ & 0.045 & 0.174 & 0.601 & 0.007 & 0.376 \\
\hline & MT & NP & $6.1 \pm 0.0$ & $58.0 \pm 4.8$ & $2.3 \pm 0.3$ & $0.65 \pm 0.00 \mathrm{a}$ & $614.4 \pm 16.3$ \\
\hline & & RP30 & $6.3 \pm 0.1$ & $60.5 \pm 3.7$ & $2.4 \pm 0.2$ & $0.53 \pm 0.01 b$ & $621.4 \pm 44.5$ \\
\hline & & RP60 & $6.3 \pm 0.1$ & $572 \pm 2.3$ & $2.2 \pm 0.2$ & $0.53 \pm 0.02 b$ & $754.1 \pm 72.8$ \\
\hline & & $p$ & 0.361 & 0.813 & 0.874 & 0.001 & 0.166 \\
\hline & HT & $\mathrm{NP}$ & $6.2 \pm 0.2$ & $45.4 \pm 2.8$ & $2.6 \pm 0.5$ & $0.59 \pm 0.02 \mathrm{a}$ & $694.3 \pm 33.3$ \\
\hline & & RP30 & $6.1 \pm 0.2$ & $57.2 \pm 3.7$ & $2.9 \pm 0.2$ & $0.47 \pm 0.02 \mathrm{~b}$ & $616.2 \pm 27.0$ \\
\hline & & RP60 & $6.0 \pm 0.1$ & $54.4 \pm 2.1$ & $2.7 \pm 0.2$ & $0.53 \pm 0.02 \mathrm{ab}$ & $719.8 \pm 10.9$ \\
\hline & & $p$ & 0.664 & 0.068 & 0.809 & 0.030 & 0.070 \\
\hline
\end{tabular}

CK, control site; LT, light thinning; MT, moderate thinning; HT, high thinning. NP, natural precipitation; RP30, 30\% reduced precipitation; RP60, 60\% reduced precipitation. TC, total carbon; TN, total nitrogen; TP, total phosphorus; MBC, microbial biomass carbon. Data (mean \pm standard error, $n=3$ ) followed by different letters within a column differ significantly according to Tukey's test $(p<0.05)$. When the difference is not statistically significant, no letter notation is given. 
Table 2. Soil phosphorus fractions in the dry and rainy seasons under different precipitation and thinning treatments in L. principis-rupprechtii plantations.

\begin{tabular}{|c|c|c|c|c|c|c|c|c|c|c|c|}
\hline \multirow{2}{*}{ Season } & \multirow{2}{*}{ Thinning } & \multirow{2}{*}{ Precipitation } & $\mathbf{R}-\mathbf{P}_{\mathbf{i}}$ & Bic-P $_{i}$ & Bic-Po & $\mathrm{OH}-\mathrm{P}_{\mathrm{i}}$ & OH-P & Dil.HCl-P ${ }_{i}$ & Conc.HCl-P ${ }_{i}$ & Conc.HCl-P & Res- $P_{i}$ \\
\hline & & & \multicolumn{9}{|c|}{$\mathrm{mg} \mathrm{kg}^{-1}$} \\
\hline \multirow[t]{16}{*}{ Dry } & CK & $\mathrm{NP}$ & $6.5 \pm 0.1$ & $7.7 \pm 0.5$ & $5.5 \pm 0.5 \mathrm{a}$ & $10.8 \pm 1.6$ & $76.8 \pm 5.4$ & $38.8 \pm 2.2 \mathrm{a}$ & $46.7 \pm 2.8 \mathrm{a}$ & $83.8 \pm 1.0 \mathrm{a}$ & $61.0 \pm 3.7$ \\
\hline & & RP30 & $7.1 \pm 1.1$ & $7.8 \pm 0.7$ & $3.1 \pm 0.1 \mathrm{~b}$ & $11.3 \pm 0.2$ & $65.7 \pm 3.4$ & $25.2 \pm 1.1 \mathrm{~b}$ & $29.9 \pm 1.6 b$ & $71.1 \pm 1.1 \mathrm{~b}$ & $64.2 \pm 4$ \\
\hline & & RP60 & $6.9 \pm 0.5$ & $6.7 \pm 0.0$ & $3.0 \pm 0.1 \mathrm{~b}$ & $12.5 \pm 1.1$ & $87.7 \pm 10.2$ & $35.1 \pm 1.1 \mathrm{a}$ & $54.5 \pm 3.8 \mathrm{a}$ & $45.2 \pm 3.1 \mathrm{c}$ & $64.2 \pm 8$ \\
\hline & & $p$ & 0.850 & 0.323 & 0.002 & 0.577 & 0.163 & 0.002 & 0.002 & $<0.001$ & 0.900 \\
\hline & LT & $\mathrm{NP}$ & $6.1 \pm 1.0$ & $7.0 \pm 1.1$ & $14.1 \pm 0.2 \mathrm{a}$ & $11.8 \pm 2.5$ & $87.6 \pm 7.4$ & $40.0 \pm 5.7$ & $43.6 \pm 3.1$ & $114.8 \pm 9.5 \mathrm{a}$ & $85.5 \pm 21.2$ \\
\hline & & RP30 & $10.5 \pm 0.6$ & $7.5 \pm 0.3$ & $15.9 \pm 1.5 \mathrm{a}$ & $11.6 \pm 0.8$ & $77.6 \pm 4.3$ & $34.8 \pm 2.4$ & $38.3 \pm 1.6$ & $66.2 \pm 6.2 \mathrm{~b}$ & $86.8 \pm 0.5$ \\
\hline & & RP60 & $9.3 \pm 1.7$ & $7.7 \pm 1.2$ & $7.0 \pm 1.4 \mathrm{~b}$ & $12.8 \pm 0.5$ & $92.3 \pm 2.2$ & $37.4 \pm 2.7$ & $43.6 \pm 1.7$ & $59.3 \pm 3.0 \mathrm{~b}$ & $97.8 \pm 7.4$ \\
\hline & & $p$ & 0.087 & 0.894 & 0.004 & 0.839 & 0.199 & 0.654 & 0.236 & 0.002 & 0.771 \\
\hline & MT & $\mathrm{NP}$ & $13.4 \pm 0.9$ & $11.6 \pm 2.7$ & $21.7 \pm 4.7 \mathrm{a}$ & $17.4 \pm 2.1$ & $167.1 \pm 20.1$ & $84.8 \pm 8.9$ & $30.9 \pm 2.0$ & $132.0 \pm 8.9 \mathrm{a}$ & $155.2 \pm 14.1 \mathrm{a}$ \\
\hline & & RP30 & $11.7 \pm 0.3$ & $14.2 \pm 1.3$ & $17.0 \pm 1.2 \mathrm{ab}$ & $17.3 \pm 1.1$ & $137.1 \pm 26.2$ & $73.6 \pm 18.5$ & $24.5 \pm 2.1$ & $115.7 \pm 2.8 \mathrm{ab}$ & $115.0 \pm 7.5 \mathrm{ab}$ \\
\hline & & RP60 & $12.6 \pm 1.3$ & $11.6 \pm 3.0$ & $8.9 \pm 0.9 \mathrm{~b}$ & $16.3 \pm 1.5$ & $115.2 \pm 4.3$ & $62.4 \pm 5.6$ & $33.7 \pm 3.7$ & $95.4 \pm 5.1 \mathrm{~b}$ & $87.8 \pm 11.1 \mathrm{~b}$ \\
\hline & & $p$ & 0.509 & 0.702 & 0.049 & 0.872 & 0.240 & 0.483 & 0.125 & 0.016 & 0.015 \\
\hline & HT & $\mathrm{NP}$ & $10.2 \pm 2.8$ & $10.0 \pm 1.1$ & $14.4 \pm 4.6$ & $10.8 \pm 0.2$ & $125.8 \pm 8.1$ & $66.7 \pm 16.1$ & $40.1 \pm 2.2$ & $76.7 \pm 3.8$ & $100.7 \pm 15.3$ \\
\hline & & RP30 & $12.1 \pm 0.9$ & $12.3 \pm 1.3$ & $18.2 \pm 1.9$ & $12.3 \pm 0.6$ & $118.7 \pm 4.3$ & $58.3 \pm 10.4$ & $37.0 \pm 1.3$ & $66.7 \pm 2.0$ & $93.6 \pm 2.9$ \\
\hline & & RP60 & $12.8 \pm 1.0$ & $9.6 \pm 0.5$ & $9.3 \pm 0.8$ & $14.4 \pm 1.9$ & $115.2 \pm 17.0$ & $75.6 \pm 2.4$ & $42.1 \pm 1.0$ & $65.6 \pm 2.8$ & $80.6 \pm 2.0$ \\
\hline & & $p$ & 0.602 & 0.195 & 0.174 & 0.185 & 0.798 & 0.577 & 0.154 & 0.069 & 0.350 \\
\hline \multirow[t]{16}{*}{ Rainy } & CK & $\mathrm{NP}$ & $10.3 \pm 0.7$ & $8.4 \pm 0.2$ & $10.1 \pm 2.7 \mathrm{ab}$ & $12.3 \pm 0.0 \mathrm{~b}$ & $135.2 \pm 0.8 \mathrm{a}$ & $51.8 \pm 0.1$ & $31.2 \pm 0.1$ & $96.7 \pm 3.7 \mathrm{a}$ & $155.9 \pm 8.5$ \\
\hline & & RP30 & $7.4 \pm 0.9$ & $9.4 \pm 1.3$ & $15.0 \pm 0.2 \mathrm{a}$ & $14.1 \pm 0.5 \mathrm{a}$ & $83.1 \pm 9.2 \mathrm{~b}$ & $42.7 \pm 5.5$ & $28.5 \pm 2.7$ & $88.8 \pm 2.3 \mathrm{a}$ & $150.3 \pm 12.5$ \\
\hline & & RP60 & $7.4 \pm 0.5$ & $7.7 \pm 0.0$ & $6.5 \pm 0.5 \mathrm{~b}$ & $10.3 \pm 0.2 \mathrm{c}$ & $83.1 \pm 2.3 \mathrm{~b}$ & $39.1 \pm 5.1$ & $28.9 \pm 1.2$ & $50.0 \pm 2.4 \mathrm{~b}$ & $150.2 \pm 5.6$ \\
\hline & & $p$ & 0.063 & 0.330 & 0.025 & $<0.001$ & 0.001 & 0.181 & 0.519 & $<0.001$ & 0.885 \\
\hline & LT & $\mathrm{NP}$ & $10.6 \pm 1.0$ & $9.4 \pm 0.7$ & $10.5 \pm 1.3$ & $21.7 \pm 0.1$ & $129.7 \pm 4.2 \mathrm{a}$ & $61.7 \pm 2.4$ & $32.8 \pm 1.0$ & $128.3 \pm 5.9 \mathrm{a}$ & $162.6 \pm 10.2$ \\
\hline & & RP30 & $7.5 \pm 0.6$ & $6.2 \pm 0.7$ & $15.0 \pm 2.8$ & $20.2 \pm 0.1$ & $92.0 \pm 9.9 b$ & $52.3 \pm 5.5$ & $39.6 \pm 5.1$ & $74.1 \pm 6.2 \mathrm{~b}$ & $164.8 \pm 10.8$ \\
\hline & & RP60 & $8.7 \pm 0.8$ & $7.5 \pm 0.8$ & $10.8 \pm 0.6$ & $19.4 \pm 1.3$ & $75.1 \pm 1.5 b$ & $55.5 \pm 7.4$ & $44.6 \pm 2.0$ & $79.0 \pm 2.1 \mathrm{~b}$ & $163.1 \pm 11.3$ \\
\hline & & $p$ & 0.090 & 0.066 & 0.226 & 0.173 & 0.002 & 0.505 & 0.100 & $<0.001$ & 0.989 \\
\hline & MT & $\mathrm{NP}$ & $10.9 \pm 1.2$ & $8.5 \pm 0.7$ & $18.5 \pm 1.7 \mathrm{a}$ & $20.4 \pm 0.2$ & $166.7 \pm 7.8 \mathrm{a}$ & $105.9 \pm 9.8$ & $31.6 \pm 0.3$ & $150.5 \pm 6.0 \mathrm{a}$ & $135.9 \pm 4.4$ \\
\hline & & RP30 & $12.1 \pm 2.7$ & $8.7 \pm 1.4$ & $15.7 \pm 0.6 \mathrm{a}$ & $24.9 \pm 3.9$ & $102.0 \pm 1.5 \mathrm{~b}$ & $100.7 \pm 3.5$ & $23.9 \pm 3.5$ & $108.7 \pm 2.1 \mathrm{~b}$ & $135.9 \pm 7.5$ \\
\hline & & RP60 & $15.4 \pm 1.8$ & $7.9 \pm 1.1$ & $10.1 \pm 0.4 b$ & $18.8 \pm 2.0$ & $79.7 \pm 2.1 \mathrm{c}$ & $105.3 \pm 15.8$ & $25.2 \pm 2.2$ & $121.9 \pm 4.9 \mathrm{~b}$ & $142.5 \pm 8.4$ \\
\hline & & $p$ & 0.318 & 0.874 & 0.005 & 0.288 & $<0.001$ & 0.935 & 0.122 & 0.002 & 0.754 \\
\hline & HT & $\mathrm{NP}$ & $13.1 \pm 1.6$ & $8.3 \pm 0.8$ & $19.0 \pm 0.3 a$ & $22.0 \pm 0.2$ & $123.2 \pm 5.4 \mathrm{a}$ & $83.6 \pm 8.9$ & $31.2 \pm 0.3$ & $131.5 \pm 18.9$ & $159.7 \pm 10.9$ \\
\hline & & RP30 & $11.3 \pm 2.5$ & $8.1 \pm 1.4$ & $13.3 \pm 0.8 \mathrm{~b}$ & $20.4 \pm 1.8$ & $98.5 \pm 6.3 \mathrm{~b}$ & $50.0 \pm 9.3$ & $32.3 \pm 8.6$ & $90.3 \pm 6.3$ & $149.5 \pm 6.9$ \\
\hline & & RP60 & $9.5 \pm 1.3$ & $10.5 \pm 2.5$ & $8.8 \pm 0.9 c$ & $19.7 \pm 0.9$ & $100.7 \pm 2.6 \mathrm{~b}$ & $77.8 \pm 10.6$ & $39.8 \pm 4.4$ & $109.4 \pm 8.7$ & $155.5 \pm 5.5$ \\
\hline & & $p$ & 0.442 & 0.580 & $<0.001$ & 0.424 & 0.024 & 0.098 & 0.533 & 0.146 & 0.684 \\
\hline
\end{tabular}

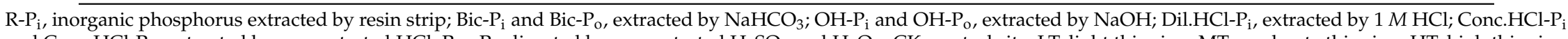
and Conc.HCl- $\mathrm{P}_{\mathrm{o}}$, extracted by concentrated $\mathrm{HCl}$; Res- $\mathrm{P}_{\mathrm{i}}$; digested by concentrated $\mathrm{H}_{2} \mathrm{SO}_{4}$ and $\mathrm{H}_{2} \mathrm{O}_{2}$. CK, control site; LT, light thinning; $\mathrm{MT}$, moderate thinning; $\mathrm{HT}$, high thinning. NP, natural precipitation; RP30, 30\% reduced precipitation; RP60, 60\% reduced precipitation. Data (mean \pm standard error, $n=3$ ) followed by different letters within a column differ significantly according to Tukey's test $(p<0.05)$. When the difference is not statistically significant, no letter notation is given. 
Table 3. Coefficient (r) for the Pearson's correlation between soil acid phosphomonoesterase (AcPME) and soil properties in L. principis-rupprechtii plantations.

\begin{tabular}{|c|c|c|c|c|c|c|c|c|c|c|c|c|c|c|}
\hline Soil Properties & $\mathrm{pH}$ & TC & TN & TP & MBC & $\mathbf{R}-\mathbf{P}_{\mathbf{i}}$ & Bic-P $P_{i}$ & Bic-P & $\mathrm{OH}-\mathrm{P}_{\mathbf{i}}$ & $\mathrm{OH}-\mathrm{P}_{\mathrm{o}}$ & Dil.HCl-P $_{\mathbf{i}}$ & Conc.HCl-P ${ }_{i}$ & Conc.HCl-P & $\operatorname{Res} P_{i}$ \\
\hline AcPME & 0.24 & 0.15 & $-0.54^{* *}$ & $0.71^{* *}$ & $0.77^{* *}$ & $0.35^{* *}$ & 0.05 & $0.46^{* *}$ & $0.64^{* *}$ & $0.33^{* *}$ & $0.56^{* *}$ & $-0.48^{* *}$ & $0.58^{* *}$ & 0.70 * \\
\hline
\end{tabular}

${ }^{*} p<0.05 ; * * p<0.01$. 


\subsection{Soil AcPME Activity}

Soil AcPME activity was significantly higher in the rainy season than in the dry season $(p<0.01)$, almost 1.9 times more so (Figure 2). Throughout the two seasons, soil AcPME activity levels in the NP treatment were as follows, in decreasing order: HT $\left(447.2 \pm 50.8 \mu \mathrm{g} \mathrm{g}^{-1} \mathrm{~h}^{-1}\right)$, MT $\left(441.2 \pm 49.6 \mu \mathrm{g} \mathrm{g}^{-1} \mathrm{~h}^{-1}\right)$, CK $\left(390.9 \pm 64.1 \mu \mathrm{g} \mathrm{g}^{-1} \mathrm{~h}^{-1}\right)$ and LT $\left(376.3 \pm 55.6 \mu \mathrm{g} \mathrm{g}^{-1} \mathrm{~h}^{-1}\right)$.

The effect of precipitation on soil AcPME activity varied with season and thinning treatment (Figure 2). In the dry season, the NP treatment was most conducive to improving soil AcPME activity in all four thinning scenarios (Figure 2a). Specifically, for CK and LT, the NP treatment resulted in significantly greater activity than RP30 ( $p=0.004$ and 0.036$)$ and RP60 $(p=0.003$ and 0.012$)$ respectively. As for MT and HT, although there was no significant difference in soil AcPME activity among precipitation treatments, they still displayed a downward trend with precipitation decline. The descending order of treatments for observed soil AcPME activity in MT was NP $\left(336.9 \pm 31.3 \mu \mathrm{g} \mathrm{g}^{-1} \mathrm{~h}^{-1}\right)>\mathrm{RP} 30$ $\left(323.6 \pm 6.8 \mu \mathrm{g} \mathrm{g}^{-1} \mathrm{~h}^{-1}\right)>\operatorname{RP} 60\left(256.9 \pm 1.6 \mu \mathrm{g} \mathrm{g}^{-1} \mathrm{~h}^{-1}\right)$. In HT, soil AcPME activity decreased by $11.1 \%$ and $29.3 \%$ in RP30 and RP60, respectively, compared to NP.

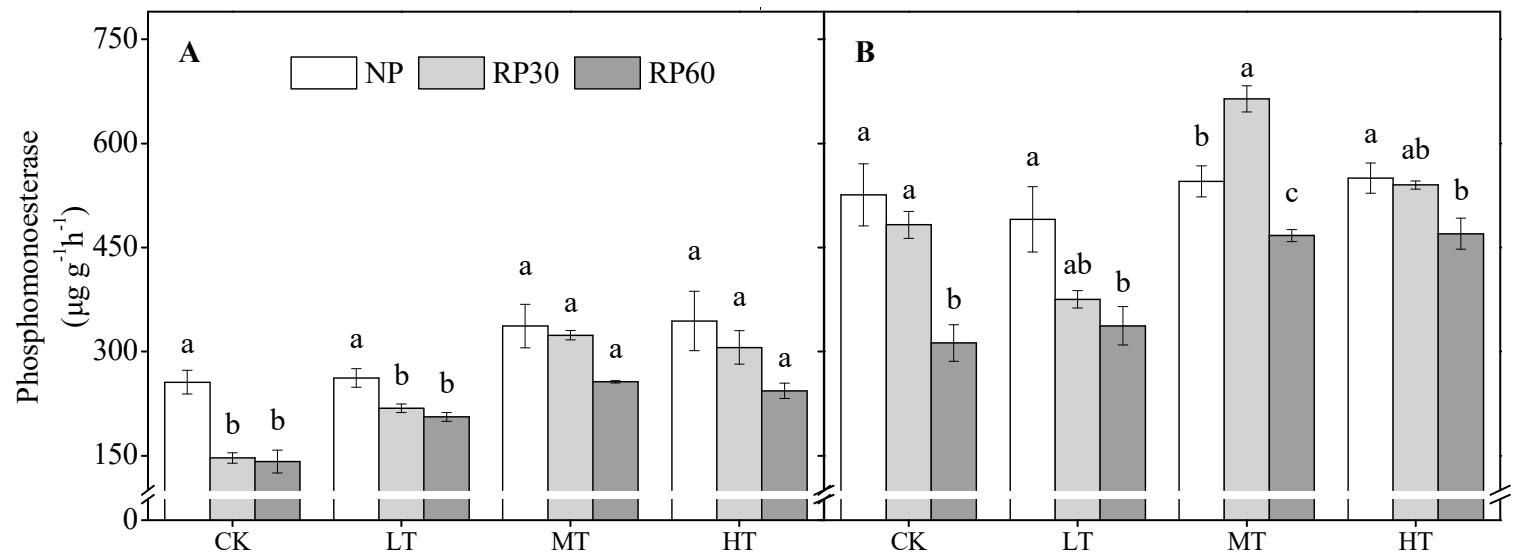

Figure 2. Soil acid phosphomonoesterase (AcPME) activity in the dry (A) and rainy (B) seasons under different precipitation and thinning treatments in L. principis-rupprechtii plantations. CK, control site; LT, light thinning; MT, moderate thinning; HT, high thinning. NP, natural precipitation; RP30, 30\% reduced precipitation; RP60, 60\% reduced precipitation. Error bars indicate standard error $(n=3)$. Different lowercase letters denote significant differences between precipitation treatments according to Tukey's test $(p<0.05)$.

In the rainy season, soil AcPME activity decreased significantly in RP60 compared with NP (Figure 2b). Specifically, soil AcPME activity in CK, LT, MT and HT were 40.6\%, 31.3\%, 14.4\% and $14.6 \%$ lower, respectively, for the RP60 than the NP treatment. However, with the exception of MT, soil AcPME activity did not differ significantly between the RP30 and NP treatments. In contrast in MT, soil AcPME activity increased significantly in the RP30 treatment (Figure 2b). Among the four thinning scenarios, the responses of soil AcPME activity to precipitation were the most pronounced in MT. The activity in all three precipitation treatments differed significantly in MT.

\section{Discussion}

\subsection{Soil AcPME Activity in the Dry and Rainy Seasons}

Consistent with the hypothesis, our study found that soil AcPME activity was significantly higher in the rainy season than the dry season (Figure 2). Higher soil moisture (Figure 1) and MBC (Table 1) were observed in the rainy season. The higher AcPME activity during the rainy season could be partly explained by the relatively high $\mathrm{MBC}$ and soil moisture content, which together promoted $\mathrm{P}_{\mathrm{o}}$ mineralization [39]. To some extent, this trend is in line with another study conducted in a desert 
steppe of northwestern China [40] and the southern USA [20], where the authors found that soil AcPME activities were significantly stimulated by regimes of increasing precipitation. They proposed that precipitation significantly affects the soil microbial biomass, thereby changing the activity of soil extracellular enzymes involved in P (AcPME) metabolism. Huang, et al. [41] also found that soil phosphatase activity increased significantly during the rainy season, which might have been a response to the increasing $\mathrm{P}$ demand caused by the vigorous growth of plants and strong microbial activity. On the contrary, the dry season was not favorable for microbial growth and activity and was the low-point for biological activity [42]. This conclusion is in line with most experiments studying enzymatic production under different levels of water availability, which tend to show lowered phosphatase activity under drought conditions [43].

\subsection{Effects of Thinning on Soil AcPME Activity under Natural Levels of Precipitation}

Our results showed that MT and HT exhibited higher soil AcPME activities than CK and LT. Both soil microenvironment and nutrient status of the L. principis-rupprechtii plantations were recognized as the major contribution to soil AcPME activity, after thinning treatment [44,45]. In our study, soil chemical properties were closely related to soil AcPME activity (Table 3), and the soil nutrient conditions of MT and HT were superior to those of CK and LT (Supplementary Table S2 and Figure S3). This was also partly supported by other studies $[12,46,47]$, which suggests that the better the soil nutrient condition, the higher the soil AcPME activity should be. In addition, MT and HT had a soil microenvironment (high soil moisture) that is more conducive to microbial growth [31], as well as higher soil microbial biomass (Table 1) [48] and microbial community function [49]. This also contributed to high soil AcPME activity because this enzyme is partly secreted from microbes [50,51]. Moreover, Renella et al. [52] indicates that microbial production of AcPME was higher in forest soil with a $\mathrm{pH}$ of 5.1-7.

\subsection{Effects of Precipitation Treatments on Soil AcPME Activity}

In the dry season, the NP treatment was more conducive to improving soil AcPME activity in all thinning scenarios, but precise effects differed among the different degrees of thinning (Figure 2a). In CK and LT, which had low soil moisture, soil AcPME activity was greatest in the NP treatment, indicating that water was an important regulator of soil $\mathrm{P}$ transformation in these two plantations [53]. Tian, et al. [31] reported that the seasonal precipitation in the spring is insufficient to meet the exuberant growth potential of plants. Thus, the RP treatments potentially inhibited plant growth, thereby reducing the $P$ demand $[23,24]$. This could also be attributed to the decreased activity of microorganisms under low moisture conditions, which results in limited $P_{o}$ mineralization $[1,20]$. Furthermore, in the dry season, concentrations of TP, MBC and Bic- $\mathrm{P}_{\mathrm{o}}$ were significantly higher in NP than RP treatments (Tables 1 and 2). A similar result was obtained by Hou, et al. [54], who believed that the increase in soil AcPME activity may be a combined result of the higher $\mathrm{MBC}$ and the easily mineralizable labile $\mathrm{P}_{\mathrm{o}}$. Bhandari, et al. [20] also found that the higher enzyme activities including $P$ mineralization was associated with higher levels of SOC, TN, MBC, and MBN. In MT and HT, precipitation treatments had no significant effect on soil AcPME activity, probably due to their well-established self-regulation mechanism [55].

In the rainy season, soil AcPME activities showed different response patterns to precipitation treatments under different thinning scenarios. Soil AcPME activity and soil moisture were lower in RP60 than in NP treatments under all thinning scenarios (Figure 2b and Figure S2). Other studies have also found similar results reported, which indicate that soil moisture is a major driving factor of phosphatase activity [12,25]. Our results show that in the rainy season, RP60 treatments significantly reduced Bio- $\mathrm{P}_{\mathrm{o}}, \mathrm{OH}-\mathrm{P}_{\mathrm{o}}$ and Conc.HCl- $\mathrm{P}_{\mathrm{o}}$ concentrations (Table 2), which could be one of the reasons for the decrease in soil AcPME activity. Our analysis is consistent with that of Turner and Haygarth [56], who proposed that high $\mathrm{P}_{\mathrm{o}}$ in soil represent the most useful $\mathrm{P}$ fractions for predicting potential phosphatase activity. $P_{o}$ fractions account for labile, moderately labile, and non-labile forms $[57,58]$, but the fraction 
overall is a suitable indicator of the ability of soil-plant system to obtain labile P (mineralization) because it is a natural potential substrate for phosphatase [12]. This reinforces the idea that all three forms are needed to better predict the P cycle capacity of forest ecosystems. In addition, in LT, the RP60 treatment led to further acidification of the soil (Table 1), which might also be a factor causing the decline of soil AcPME activity. When the soil $\mathrm{pH}$ value is relatively low, considerable aluminum toxicity occurs, which inhibits plant root growth and nutrient absorption $[59,60]$. These factors reduce soil AcPME activity, which is closely related to microbe and root growth [61,62]. The decrease of soil acid phosphatase activity caused by drought would lead to the decrease of P availability for plants, which would lead to long-term competition for P in regional forest ecosystems.

However, in the rainy season, the RP30 treatment did not inhibit soil AcPME activity, and even promoted it in MT. This is attributed to the fact that the abundant natural precipitation met the water demand of plant growth during the rainy season, though excessive precipitation is not advantageous for plants in a way that it may inhibit the mineralization potential of soil $\mathrm{P}_{\mathrm{o}}$ [63]. Because soil moisture was already high in MT (Figure 1), excessive natural precipitation was not conducive to the diffusion of oxygen in the soil $[64,65]$, which may have caused soil hypoxia, thus limiting plant root growth and soil microbial activity [66]. Therefore, when precipitation is appropriately reduced (i.e., RP30), the soil hypoxic environment is alleviated, thereby increasing soil AcPME activity, as in the RP30 treatment of MT. This would also improve the mineralization capacity of soil $\mathrm{P}_{\mathrm{O}}$.

\section{Conclusions}

Driven by the seasonality of precipitation, soil acid phosphomonoesterase (AcPME) activities showed an obvious seasonal pattern. That is, soil AcPME activity was significantly higher in the rainy season, which is associated with higher microbial activity and increased $\mathrm{P}$ demand, than in the dry season. Soil AcPME activity gradually increased with thinning intensity. That is, high thinning (HT) and moderate thinning (MT) were higher than light thinning (LT) and control (CK), indicating that HT and MT had good soil conditions. Our results also indicate that a reasonable precipitation distribution is an important factor in controlling the soil P cycle in a L. principis-rupprechtii plantation. In the dry season, the natural precipitation (NP) treatment was more conducive to improving soil AcPME activity for all thinning scenarios, which attribute to increasing microbial biomass carbon $(\mathrm{MBC})$ and easily mineralizable labile $\mathrm{P}_{\mathrm{o}}$. In the rainy season, soil AcPME activity was significantly lower in the $60 \%$ reduced precipitation (RP60) treatment than in the NP treatment under all thinning scenarios. These influences of precipitation on soil AcPME are likely driven by RP60 effects on $\mathrm{P}_{\mathrm{O}}$ fractions (including Bio- $\mathrm{P}_{\mathrm{O}}, \mathrm{OH}-\mathrm{P}_{\mathrm{o}}$ and Conc.HCl- $\mathrm{P}_{\mathrm{O}}$ ) and further acidification of the soil. The 30\% reduced precipitation (RP30) treatment did not inhibit soil AcPME activity, and even promoted it in MT. In summary, our results indicate that a year-long drought would not be conducive to the transformation of soil $\mathrm{P}_{\mathrm{O}}$ in the forest ecosystem, but an increase in rainfall during the rainy season would also be detrimental to the mineralization of soil $\mathrm{P}_{\mathrm{o}}$ in MT L. principis-rupprechtii plantations of North China.

Supplementary Materials: The following are available online at http://www.mdpi.com/1999-4907/10/9/734/s1, Table S1: Information about the study sites under the four thinning treatments in 2014. Table S2: Repeated-measures ANOVA results for soil properties under the four thinning treatments in L. principis-rupprechtii plantations. Figure S1: Soil moisture in the dry season under natural precipitation (NP) treatments in L. principis-rupprechtii plantations following thinning. Figure S2: Soil moisture under different precipitation treatments in L. principisrupprechtii plantations. Figure S3: Soil phosphorus fractions under natural precipitation (NP) treatments in L. principis-rupprechtii plantations following thinning.

Author Contributions: H.T. Analyzed the data and wrote the manuscript. X.C. Revised the manuscript. H.H. Designed the study.

Funding: This study was supported by the National Key Research and Development Program of China (2016YFD0600205); the National Natural Science Foundation of China (31700372).

Acknowledgments: We gratefully acknowledge the support from the Taiyue Forestry Bureau and the Haodifang Forestry Centre for fieldworks. We would also like to thank Elizabeth Tokarz at the Yale University for her assistance with English language and grammatical editing of the manuscript. 
Conflicts of Interest: The authors declare no conflict of Interest.

\section{References}

1. Weihrauch, C.; Opp, C. Ecologically relevant phosphorus pools in soils and their dynamics: The story so far. Geoderma 2018, 325, 183-194. [CrossRef]

2. Peltzer, D.A.; Wardle, D.A.; Allison, V.J.; Baisden, W.T.; Bardgett, R.D.; Chadwick, O.A.; Condron, L.M.; Parfitt, R.L.; Porder, S.; Richardson, S.J. Understanding ecosystem retrogression. Ecol. Monogr. 2010, 80, 509-529. [CrossRef]

3. Sullivan, B.W.; Nifong, R.L.; Nasto, M.K.; Alvarez-Clare, S.; Dencker, C.; Soper, F.M.; Shoemaker, K.T.; Ishida, F.Y.; Zaragoza-Castells, J.; Davidson, E.A. Biogeochemical recuperation of lowland tropical forest during succession. Ecology 2019, 100, e02641. [CrossRef] [PubMed]

4. Walker, T.W.; Syers, J.K. The fate of phosphorus during pedogenesis. Geoderma 1976, 15, 1-19. [CrossRef]

5. Oberson, A.; Friesen, D.K.; Rao, I.M.; Bühler, S.; Frossard, E. Phosphorus Transformations in an Oxisol under contrasting land-use systems: The role of the soil microbial biomass. Plant Soil 2001, 237, 197-210. [CrossRef]

6. Bhandari, K.B.; West, C.P.; Longing, S.D.; Brown, C.P.; Green, P.E.; Barkowsky, E. Pollinator abundance in semiarid pastures as affected by forage species. Crop Sci. 2018, 58, 2665. [CrossRef]

7. Bhandari, K.B.; West, C.P.; Longing, S.D.; Brown, C.P.; Green, P.E. Comparison of arthropod communities among different forage types on the texas high plains using pitfall traps. Crop Forage Turfgrass Manag. 2018, 4. [CrossRef]

8. Bhandari, K.B.; West, C.P.; Klein, D.; Subbiah, S.; Surowiec, K. Essential oil composition of 'WW-B.Dahl' old world bluestem (Bothriochloa bladhii) grown in the Texas High Plains. Ind. Crops Prod. 2019, 133, 1-9. [CrossRef]

9. Turner, B.L.; Lambers, H.; Condron, L.M.; Cramer, M.D.; Leake, J.R.; Richardson, A.E.; Smith, S.E. Soil microbial biomass and the fate of phosphorus during long-term ecosystem development. Plant Soil 2013, 367, 225-234. [CrossRef]

10. Khan, K.S.; Joergensen, R.G. Response of white mustard (Sinapis alba) and the soil microbial biomass to P and $\mathrm{Zn}$ addition in a greenhouse pot experiment. J. Plant Nutr. Soil Sci. 2015, 178, 834-840. [CrossRef]

11. Tian, J.; Wei, K.; Condron, L.M.; Chen, Z.; Xu, Z.; Chen, L. Impact of land use and nutrient addition on phosphatase activities and their relationships with organic phosphorus turnover in semi-arid grassland soils. Biol. Fertil. Soils 2016, 52, 675-683. [CrossRef]

12. Margalef, O.; Sardans, J.; Fernández-Martínez, M.; Molowny-Horas, R.; Janssens, I.A.; Ciais, P.; Goll, D.; Richter, A.; Obersteiner, M.; Asensio, D. Global patterns of phosphatase activity in natural soils. Sci. Rep. 2017, 7, 1337. [CrossRef] [PubMed]

13. Marklein, A.R.; Houlton, B.Z. Nitrogen inputs accelerate phosphorus cycling rates across a wide variety of terrestrial ecosystems. New Phytol. 2012, 193, 696. [CrossRef] [PubMed]

14. Krämer, S.; Green, D.M. Acid and alkaline phosphatase dynamics and their relationship to soil microclimate in a semiarid woodland. Soil Biol. Biochem. 2000, 32, 179-188. [CrossRef]

15. Nannipieri, P.; Giagnoni, L.; Landi, L.; Renella, G. Role of Phosphatase Enzymes in Soil. In Phosphorus in Action; Springer: Lindau, ZH, Switherland, 2011; pp. 215-243.

16. Pachauri, K.; Meyer, A. Climate change 2014: Synthesis Report. Environ. Policy Collect. 2014, $27,408$.

17. Thomey, M.L.; Collins, S.L.; Vargas, R.; Johnson, J.E.; Brown, R.F.; Natvig, D.O.; Friggens, M.T. Effect of precipitation variability on net primary production and soil respiration in a Chihuahuan Desert grassland. Global Change Biol. 2015, 17, 1505-1515. [CrossRef]

18. Hu, B.; Yang, B.; Pang, X.; Bao, W.; Tian, G. Responses of soil phosphorus fractions to gap size in a reforested spruce forest. Geoderma 2016, 279, 61-69. [CrossRef]

19. Netzer, F.; Thöm, C.; Celepirovic, N.; Ivankovic, M.; Alfarraj, S.; Dounavi, A.; Simon, J.; Herschbach, C.; Rennenberg, H. Drought effects on C, N, and P nutrition and the antioxidative system of beech seedlings depend on geographic origin. J. Plant Nutr. Soil Sci. 2016, 179, 136-150. [CrossRef]

20. Bhandari, K.B.; West, C.P.; Acosta-Martinez, V.; Cotton, J.; Cano, A. Soil health indicators as affected by diverse forage species and mixtures in semi-arid pastures. Appl Soil Ecol 2018, 132, 179-186. [CrossRef] 
21. Grierson, P.F.; Adams, M.A. Plant species affect acid phosphatase, ergosterol and microbial P in a Jarrah (Eucalyptus marginata Donn ex Sm.) forest in south-western Australia. Soil Biol. Biochem. 2000, 32, 1817-1827. [CrossRef]

22. Garcia, C.; Hernandez, T.; Roldan, A.; Martin, A. Effect of plant cover decline on chemical and microbiological parameters under Mediterranean climate. Soil Biol. Biochem. 2002, 34, 635-642. [CrossRef]

23. Sardans, J.; Peñuelas, J.; Estiarte, M. Seasonal patterns of root-surface phosphatase activities in a Mediterranean shrubland. Responses to experimental warming and drought. Biol. Fertil. Soils 2007, 43, 779-786. [CrossRef]

24. Sardans, J.; Peñuelas, J. Drought decreases soil enzyme activity in a Mediterranean Quercus ilex L. forest. Soil Biol. Biochem. 2005, 37, 455-461. [CrossRef]

25. Sardans, J.; Peñuelas, J.; Ogaya, R. Experimental drought reduced acid and alkaline phosphatase activity and increased organic extractable P in soil in a Quercus ilex Mediterranean forest. Eur. J. Soil Biol. 2008, 44, 509-520. [CrossRef]

26. Turner, B.L.; Condron, L.M. Pedogenesis, nutrient dynamics, and ecosystem development: The legacy of T.W. Walker and J.K. Syers. Plant Soil 2013, 367, 1-10. [CrossRef]

27. Hedo de Santiago, J.; Lucas-Borja, M.E.; Wic-Baena, C.; Andrés-Abellán, M.; de las Heras, J. Effects of Thinning and Induced Drought on Microbiological Soil Properties and Plant Species Diversity at Dry and Semiarid Locations. Land Degrad. Dev. 2016, 27, 1151-1162. [CrossRef]

28. Yuan, J.; Jose, S.; Hu, Z.; Pang, J.; Hou, L.; Zhang, S. Biometric and Eddy Covariance Methods for Examining the Carbon Balance of a Larix principis-rupprechtii Forest in the Qinling Mountains, China. Forests 2018, 9, 67. [CrossRef]

29. Lun, F.; Liu, Y.; He, L.; Yang, L.; Liu, M.; Li, W. Life cycle research on the carbon budget of the Larix principis-rupprechtii plantation forest ecosystem in North China. J. Clean. Product. 2018, 177, 178-186. [CrossRef]

30. Cheng, X.; Kang, F.; Han, H.; Liu, H.; Zhang, Y. Effect of thinning on partitioned soil respiration in a young Pinus tabulaeformis plantation during growing season. Agr. For. Meteorol. 2015, 214-215, 473-482. [CrossRef]

31. Tian, H.; Cheng, X.; Han, H.; Jing, H.; Liu, X.; Li, Z. Seasonal Variations and Thinning Effects on Soil Phosphorus Fractions in Larix principis-rupprechtii Mayr. Plantations. Forests 2019, 10, 172. [CrossRef]

32. Shi, X.Z.; Yu, D.S.; Xu, S.X.; Warner, E.D.; Wang, H.J.; Sun, W.X.; Zhao, Y.C.; Gong, Z.T. Cross-reference for relating Genetic Soil Classification of China with WRB at different scales. Geoderma 2010, 155, 344-350. [CrossRef]

33. Boyer, J.; Groffman, P. Bioavailability of water extractable organic carbon fractions in forest and agricultural soil profiles. Soil Biol. Biochem. 1996, 28, 783-790. [CrossRef]

34. Wu, J.; Joergensen, R.; Pommerening, B.; Chaussod, R.; Brookes, P. Measurement of soil microbial biomass C by fumigation-extraction-an automated procedure. Soil Biol. Biochem. 1990, 22, 1167-1169. [CrossRef]

35. Tabatabai, M.A.; Bremner, J.M. Use of $p$-nitrophenyl phosphate for assay of soil phosphatase activity. Soil Biol. Biochem. 1969, 1, 301-307. [CrossRef]

36. Hedley, M.J.; Stewart, J.; Chauhan, B. Changes in inorganic and organic soil phosphorus fractions induced by cultivation practices and by laboratory incubations 1. Soil Sci. Soc. Am. J. 1982, 46, 970-976. [CrossRef]

37. Tiessen, H.; Moir, J. Characterization of available P by sequential extraction. In Soil Sampling and Methods of Analysis; Carter, M.R., Gregorich, E.G., Eds.; Lewis Publishers: Boca Raton, FL, USA, 1993; pp. 75-86.

38. Murphy, J.; Riley, J.P. A modified single solution method for the determination of phosphate in natural waters. Anal. Chim. Acta 1962, 27, 31-36. [CrossRef]

39. He, Y.Q.; Zhu, Y.G.; Smith, S.E.; Smith, F.A. Interactions between soil moisture content and phosphorus supply in spring wheat plants grown in pot culture. J. Plant Nutr. 2002, 25, 913-925. [CrossRef]

40. Na, X.; Yu, H.; Wang, P.; Zhu, W.; Niu, Y.; Huang, J. Vegetation biomass and soil moisture coregulate bacterial community succession under altered precipitation regimes in a desert steppe in northwestern China. Soil Biol. Biochem. 2019, 136, 107520. [CrossRef]

41. Huang, W.; Liu, J.; Zhou, G.; Zhang, D.; Deng, Q. Effects of precipitation on soil acid phosphatase activity in three successional forests in southern China. BGeo 2011, 8, 1901-1910.

42. Settineri, G.; Mallamaci, C.; Mitrović, M.; Sidari, M.; Muscolo, A. Effects of different thinning intensities on soil carbon storage in Pinus laricio forest of Apennine South Italy. Eur. J. For. Res. 2018, 137, 131-141. [CrossRef] 
43. Sardans, J.; Peñuelas, J.; Estiarte, M. Warming and drought alter soil phosphatase activity and soil $\mathrm{P}$ availability in a Mediterranean shrubland. Plant Soil 2006, 289, 227-238. [CrossRef]

44. Ushio, M.; Kitayama, K.; Balser, T.C. Tree species effects on soil enzyme activities through effects on soil physicochemical and microbial properties in a tropical montane forest on Mt. Kinabalu, Borneo. Pedobiologia 2010, 53, 227-233. [CrossRef]

45. Kim, S.; Li, G.; Han, S.H.; Kim, C.; Lee, S.-T.; Son, Y. Microbial biomass and enzymatic responses to temperate oak and larch forest thinning: Influential factors for the site-specific changes. Sci. Total Environ. 2019, 651, 2068-2079. [CrossRef]

46. Chen, C.R.; Condron, L.M.; Davis, M.R.; Sherlock, R.R. Seasonal changes in soil phosphorus and associated microbial properties under adjacent grassland and forest in New Zealand. For. Ecol. Manag. 2003, 177, 539-557. [CrossRef]

47. Chen, C.R.; Condron, L.M.; Xu, Z.H. Impacts of grassland afforestation with coniferous trees on soil phosphorus dynamics and associated microbial processes: A review. For. Ecol. Manag. 2008, 255, 396-409. [CrossRef]

48. Ma, J.; Kang, F.; Cheng, X.; Han, H. Moderate thinning increases soil organic carbon in Larix principis-rupprechtii ( Pinaceae ) plantations. Geoderma 2018, 329, 118-128. [CrossRef]

49. Wu, R.; Cheng, X.; Han, H. The Effect of Forest Thinning on Soil Microbial Community Structure and Function. Forests 2019, 10, 352. [CrossRef]

50. Souza, R.C.; Solly, E.F.; Dawes, M.A.; Graf, F.; Hagedorn, F.; Egli, S.; Clement, C.R.; Nagy, L.; Rixen, C.; Peter, M. Responses of soil extracellular enzyme activities to experimental warming and $\mathrm{CO}_{2}$ enrichment at the alpine treeline. Plant. Soil 2017, 416, 527-537. [CrossRef]

51. Yokoyama, D.; Imai, N.; Kitayama, K. Effects of nitrogen and phosphorus fertilization on the activities of four different classes of fine-root and soil phosphatases in Bornean tropical rain forests. Plant. Soil 2017, 416, 463-476. [CrossRef]

52. Renella, G.; Szukics, U.; Landi, L.; Nannipieri, P. Quantitative assessment of hydrolase production and persistence in soil. Biol. Fertility Soils 2007, 44, 321-329. [CrossRef]

53. Lang, F.; Bauhus, J.; Frossard, E.; George, E.; Kaiser, K.; Kaupenjohann, M.; Krüger, J.; Matzner, E.; Polle, A.; Prietzel, J.; et al. Phosphorus in forest ecosystems: New insights from an ecosystem nutrition perspective. J. Plant Nutr. Soil Sci. 2016, 179, 129-135. [CrossRef]

54. Hou, E.; Chen, C.; Wen, D.; Liu, X. Phosphatase activity in relation to key litter and soil properties in mature subtropical forests in China. Sci. Total Environ. 2015, 515-516, 83-91. [CrossRef]

55. Liu, C.; Jin, Y.; Liu, C.; Tang, J.; Wang, Q.; Xu, M. Phosphorous fractions in soils of rubber-based agroforestry systems: Influence of season, management and stand age. Sci. Total Environ. 2018, 616-617, 1576-1588. [CrossRef]

56. Turner, B.L.; Haygarth, P.M. Phosphatase activity in temperate pasture soils: Potential regulation of labile organic phosphorus turnover by phosphodiesterase activity. Sci. Total Environ. 2005, 344, 27-36. [CrossRef]

57. Cherubin, M.R.; Franco, A.L.; Cerri, C.E.; Karlen, D.L.; Pavinato, P.S.; Rodrigues, M.; Davies, C.A.; Cerri, C.C. Phosphorus pools responses to land-use change for sugarcane expansion in weathered Brazilian soils. Geoderma 2016, 265, 27-38. [CrossRef]

58. von Sperber, C.; Stallforth, R.; Preez, C.D.; Amelung, W. Changes in soil phosphorus pools during prolonged arable cropping in semiarid grasslands. Eur. J. Soil Sci. 2017, 68, 462-471. [CrossRef]

59. Rodrigues, M.; Pavinato, P.S.; Withers, P.J.A.; Teles, A.P.B.; Herrera, W.F.B. Legacy phosphorus and no tillage agriculture in tropical oxisols of the Brazilian savanna. Sci. Total Environ. 2016, 542, 1050-1061. [CrossRef]

60. Wang, J.; Ren, C.; Cheng, H.; Zou, Y.; Bughio, M.A.; Li, Q. Conversion of rainforest into agroforestry and monoculture plantation in China: Consequences for soil phosphorus forms and microbial community. Sci. Total Environ. 2017, 595, 769-778. [CrossRef]

61. Pii, Y.; Mimmo, T.; Tomasi, N.; Terzano, R.; Cesco, S.; Crecchio, C. Microbial interactions in the rhizosphere: Beneficial influences of plant growth-promoting rhizobacteria on nutrient acquisition process. A review. Biol. Fertility Soils 2015, 51, 403-415. [CrossRef]

62. Razavi, B.S.; Zarebanadkouki, M.; Blagodatskaya, E.; Kuzyakov, Y. Rhizosphere shape of lentil and maize: Spatial distribution of enzyme activities. Soil Biol. Biochem. 2016, 96, 229-237. [CrossRef] 
63. Reddy, K.R.; Wetzel, R.G.; Kadlec, R.H. Biogeochemistry of phosphorus in wetlands. In Phosphorus: Agriculture and the Environment; Sims, J.T., Sharpley, A.N., Eds.; American Society of Agronomy: Madison, WI, USA, 2005; pp. 263-316.

64. Davidson, E.A.; Belk, E.; Boone, R.D. Soil water content and temperature as independent or confounded factors controlling soil respiration in a temperate mixed hardwood forest. Global Change Biol. 1998, 4, $217-227$. [CrossRef]

65. Chacon, N.; Flores, S.; Gonzalez, A. Implications of iron solubilization on soil phosphorus release in seasonally flooded forests of the lower Orinoco River, Venezuela. Soil Biol. Biochem. 2006, 38, 1494-1499. [CrossRef]

66. Achat, D.L.; Augusto, L.; Bakker, M.R.; Gallet-Budynek, A.; Morel, C. Microbial processes controlling P availability in forest spodosols as affected by soil depth and soil properties. Soil Biol. Biochem. 2012, 44, 39-48. [CrossRef]

(C) 2019 by the authors. Licensee MDPI, Basel, Switzerland. This article is an open access article distributed under the terms and conditions of the Creative Commons Attribution (CC BY) license (http://creativecommons.org/licenses/by/4.0/). 